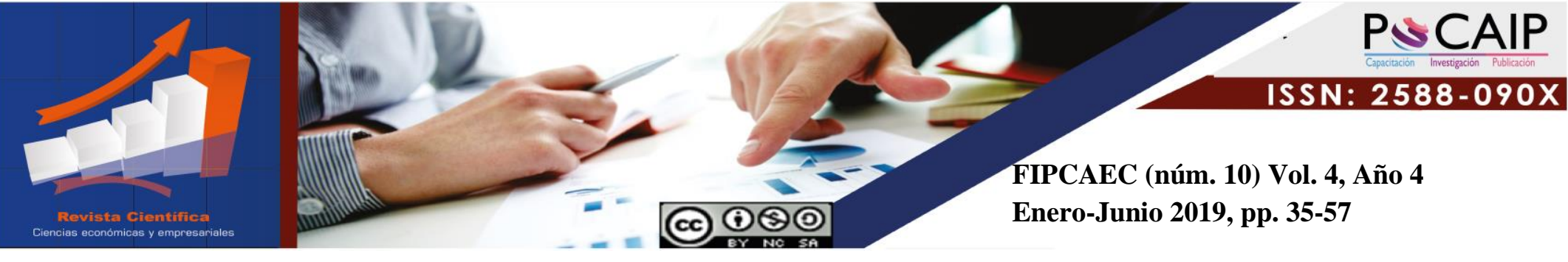

DOI: $10.23857 /$ fipcaec.v4i10.37

\title{
Importancia de la planeación estratégica en empresas en el siglo XXI
}

Importance of strategic business planning in the 21st century

Importância do planejamento estratégico de negócios no século XXI

\author{
Víctor Reinaldo Jama-Zambrano ${ }^{1}$ \\ victorreinaldojamas@hotmail.com
}

ORCID: https://orcid.org/0000-0001-7102-2352

Correspondencia: victorreinaldojamas@ hotmail.com

Recepción: 21/ 11/2018 * Aceptación: 18/12/2018 * Publicación: 15 /01/ 2019

1 Docente del Instituto Superior Pedagógico Eugenio Espejo, Chone, Ecuador. 



\title{
Resumen
}

La revisión bibliográfica relacionada con la importancia de la Planeación estratégica en las empresas durante el presente siglo, comienza por describir su definición y una breve historia de la misma, que va desde la edad antigua hasta nuestros días; son expuestos y otros mencionados algunos modelos de investigadores del tema, se revisa lo relacionado con la Administración estratégica donde se describe el análisis que debe realizar la alta gerencia para lograr los objetivos o metas de la empresa; finalmente se enuncian las tres fases de dicha preparación.

Palabras claves: Planeación estratégica; Planificación estratégica; Planeación empresarial; Administración estratégica.

\begin{abstract}
The literature review related to the importance of strategic planning in companies during the present century, begins by describing its definition and a brief history of it, which goes from the ancient age to the present day; Some models of researchers of the subject are exposed and others mentioned, the thing related to the Strategic Administration is reviewed where the analysis that the senior management must perform to achieve the objectives or goals of the company is described; finally the three phases of said preparation are enunciated.
\end{abstract}

Keywords: Strategic planning; Strategic planning; Business planning; Strategic management.

\section{Resumo}

A revisão da literatura relacionada à importância do planejamento estratégico nas empresas durante o século atual, começa descrevendo sua definição e uma breve história dela, que vai desde a era antiga até os dias atuais; Alguns modelos de pesquisadores do assunto são expostos e outros mencionados, o relacionado à Administração Estratégica, é revisto onde são descritas as análises que a alta gerência deve realizar para atingir os objetivos ou metas da empresa; finalmente, as três fases da referida preparação são enunciadas.

Palavras-chave: Planejamento estratégico; Planificação estratégica; Planejamento de negócios; Administração Estratégica 


\section{Introducción}

La planeación estratégica es función de todo gerente/director a cualquier nivel de una organización o empresa, pues actualmente la gerencia y planeación estratégica son vitales en cuanto al éxito de las compañías ya que una estrategia inadecuada puede crear serios problemas, no importando lo eficiente que puede ser una empresa internamente.

Por tanto, desde el punto de vista empresarial abordar el proceso de planeación estratégica es referido al hecho de ratificar o renunciar a determinadas áreas de negocios; al hecho de establecer la disposición de los recursos, a la posibilidad de ampliar operaciones o diversificar, y a la identificación plena de los mercados y los sujetos sociales.

Para la toma de decisiones afines con las metas y estrategias de alta dirección es necesario un programa de planeación o planificación efectivo que consiste en proporcionar una guía para los ejecutivos en todos los aspectos de un negocio entendiendo el concepto de estrategia de la siguiente manera, Cito (...): - el desarrollo y aprovechamiento de la capacidad interna para enfrentar distintos retos; - respuesta o anticipación a los cambios del medio ambiente; - la forma de competir en el mercado; - la visión de largo plazo;- el reto que resulta de preguntar: ¿En qué lugar estamos? y ¿En qué lugar deberíamos estar? (Valenzuela Argoti, 2016)

La generación de una toma de decisiones de manera estratégica es importante partir de la comprensión del conjunto de fenómenos internos y externos en que participa la empresa así como los objetivos que ésta trata de alcanzar en ese contexto; con toda su información la mente estratega examina el conjunto de esos factores, comprende y formula alternativas orientadas hacia lo que quiere que suceda el gerente estratega.

Este objetivo es logrado cuando se cuente con un instrumento de apoyo para hacer frente a los retos actuales, dada la creciente competitividad del mercado y la fuerte dinámica de cambio que se vive en este milenio, prácticamente en todos los ámbitos.

Es por ello que las organizaciones deben revisar y ajustar sus estrategias para competir en forma satisfactoria, así como para aprovechar las oportunidades o evitar las amenazas que el tiempo trae 
consigo, e incluso, ir un paso más allá al buscar cómo dar lugar a un futuro deseado y crear formas innovadoras de acción.

El calificativo de estratégico se aplica en los más diversos casos y circunstancias: - Planes de largo plazo; - recursos valiosos; programas de expansión; cómo salir de embrollos; formación de alianzas; lanzamiento de productos; reorganización de empresas; - grandes proyectos (Cendejas Valdéz, 2014)

\section{Desarrollo}

\section{Definición e historia de la planeación estratégica}

Etimológicamente la palabra estrategia proviene del Griego stategos $=$ "Un general" $=>$ "ejercito", acaudillar. Verbo griego stategos = "planificar la destrucción de los enemigos en razón eficaz de los recursos".

En la época antigua moderna y contemporánea, aparecen los científicos, tales como: Sócrates en la Grecia antigua, quien emitió su definición; en el contexto de los negocios en 1944 se comienza a aplicar en el campo económico y académico por los científicos Von Neuman y Morgenstern en su obra "La Teoría del Juego" los más significativos.

\section{La palabra planificación, según la Real Academia Española, la define de la siguiente manera:}

- Acción y efecto de planificar

- Plan general, metódicamente organizado y frecuentemente de gran amplitud, para obtener un objetivo determinado, tal como el desarrollo armónico de una ciudad, el desarrollo económico, la investigación científica, el funcionamiento de una industria, etc. (Velázquez, 2013), (Jackeline \& Posadas, 2016), (Sánchez Jacas, 2017)

\section{También esta organización define la palabra estrategia como:}

- Arte de dirigir las operaciones militares.

- Arte, traza para dirigir un asunto 
SunTzu, uno de los estrategas de la época antigua tiene su surgimiento el termino estrategia ya que influyó en las concepciones militares, este aguerrido pensador no conocía el concepto de la estrategia pero si de la ofensiva militar; definida como la capacidad que tiene el general para conseguir provecho de las circunstancias del entorno a través de sus habilidades sin la aplicación de otros procedimientos.

Aníbal con propósito de conquistar Roma, realizo una serie de procesos y actividades como el análisis del medio ambiente y como emplear adecuadamente los medios para la implementación de métodos y proyectos para el logro de los objetivos.

Baidaba y Nicolás Maquiavelo quienes trazaban el aprovechamiento de las oportunidades así como el análisis de los hechos anteriores; las causales de su éxito y fracaso; sorteando las amenazas o situaciones perjudiciales y la necesidad de la planeación para un buen gobierno.

En Grecia, Sócrates comparaba la actividad de un empresario con las de un general alegando que en toda misión o actividad quienes la ejecutan deben realizar un plan de acción explícito y emplear los recursos adecuadamente para lograr sus objetivos.

Von Neuman y Morgenstern fueron los autores pioneros que enfocaron la planeación estratégica a los negocios planteando el concepto de la realización de actos por parte de las empresas en base a una situación determinada.

A inicios de la década de los cincuenta, las firmas de negocios y más tarde otros tipos de organizaciones de producción de servicios y bienes, empezaron a preocuparse por sus desajustes con el medio ambiente; y es así como Peter Druker en el año 1954, la define como, Cito (...): “ la estrategia como la capacidad de los gerentes para analizar la situación actual de las unidades empresariales y su capacidad e aptitud para someterlas al cambio, conociendo los recursos que esta posee al igual aquellos que esta requiere para su óptimo desempeño".

Concluida la segunda guerra mundial Alfred D. Chandler en el año 1962, teniendo en cuenta las enseñanzas empresariales y el comportamiento de importantes compañías tales como General Motors, Sears, Chevron Co. y DuPont quien definió la planeación estratégica como, Cito (...) “ 
los parámetros a desarrollar y la óptima utilización de los recursos para el logro de los objetivos organizacionales"

En el año 1978, Charles H. Hofer diseñaron la administración estratégica definiéndola como, Cito (...) "un proceso integrado por un análisis en el cual se definen las metas y se establecen las estrategias y una implementación del plan estratégico basada en el control y la ejecución de éste”.

Desde la década de 1960 hasta mediados de 1980, la planeación estratégica subrayaba un enfoque de arriba abajo respecto al establecimiento de metas y planeación, es decir la alta dirección y los especialistas en planeación estratégica desarrollaban metas y planes para toda la organización; en el año 1992, Henry Mintzbergla define como, Cito (...) "una acción deliberada que puede ser desarrollada como un plan de acción, maniobra, patrón, posición y/o perspectiva”.

Como se evidencia en estas definiciones dadas por diversos expertos del tema los que coinciden parcialmente en sus reflexiones; no obstante, se pueden percibir claramente dos tendencias fundamentales; la primera se refiere a la dinámica de la empresa con su entorno; la segunda, defiende el arte de generar objetivos, programas y políticas y la forma de alcanzarlos como vía para cumplir la misión de la misma. En la actualidad se define como el proceso que permite a los directivos o las personas encargadas de la toma de decisiones en las empresas el procesamiento y análisis de la información para que estas organizaciones logren altos niveles de competitividad y desarrollo; dado a que el paso del tiempo éste concepto ha sido reestructurado por los constantes cambios del mundo producidos en parte por la globalización y el flujo constante de la información (Cendejas Valdéz, 2014). (Delgado Morato, 2012)

La ventaja competitiva es de lo que trata la estrategia, lo que la distingue de todos los demás tipos de planeación de los negocios, puesto que el único propósito de la planeación estratégica viene a ser el permitir que la compañía obtenga con la mayor eficiencia posible una ventaja sostenible sobre sus competidores, por tanto el concepto de estrategia tiene un carácter multidimensional, que gira alrededor de cinco interrogantes claves:

1. ¿En qué negocio conviene participar y cómo manejar la familia de negocios?,

2. ¿Cómo competir en cada negocio?,

3. ¿Cómo mejorar la capacidad competitiva?, 
4. ¿Qué retos se prevén?, y

5. ¿Cuál es la imagen objetivo?;

Además trata de indicar con las siguientes interrogantes: - ¿Qué hacer? - ¿Cómo hacerlo? ¿Dónde hacerlo? - ¿Quién lo va a hacer? - ¿Cuándo hacerlo?; es un proceso que señala anticipadamente cada acción o actividad que se debe realizar

Todas éstas interrogantes son respondidas a través de la planeación estratégica que son actividades relacionadas con las cuatro áreas básicas de toda empresa: dirección, producción, mercados, finanzas y esta relación depende la vida de la empresa (Valenzuela Argoti, 2016)

\section{Modelos de planeación}

En el año 1994Sallenave diseñó un modelo conceptual en que presenta una idea de lo que debería ser la planeación estratégica en general, o una efigie de algo formado mediante la generalización de particularidades, a diferencia de un modelo operativo utilizado realmente por las empresas. Existen diferentes modelos que pueden ayudar a las organizaciones a generar una planeación extremadamente exitosa, entre ellos tenemos:

1. Modelo de planeación de George Steiner. En este modelo se encuentran los propósitos socioeconómicos fundamentales de la organización de la alta gerencia y los estudios del medio ambiente.

2. Modelo de Willian Newman. Definiéndose el proceso de planeación desde el estudio de las etapas básicas de una decisión que se tome; estas son: diagnóstico del problema, determinación de soluciones optativas, pronóstico de resultados en cada acción y finalmente la elección del camino a seguir. Estas etapas se realizan de forma sucesiva.

3. Modelo de planeación de Bateman y Snell. Estos autores señalan que el proceso de planeación es un proceso dual en que dos características convergen: las etapas generales involucradas en el proceso de decisión y las etapas específicas de la planeación formal. La primera presupone todas las acciones orientadas a la identificación y precisión del problema que se quiere resolver y en la segunda se realizan las actividades formales, 
convirtiéndolas en planes y programas susceptibles para ser llevados a la práctica; fijando sus objetivos; el proceso de implementación y la evaluación correspondiente.

4. Modelo de planeación de Mintzberg y Waters. Este modelo considera que combinar el recurso humano con el trabajo del proceso de las organizaciones permiten determinar las políticas y programas para alcanzar los objetivos y las metas de las organizaciones; los autores de este modelo explican que así es posible asegurarse de las políticas para convertirse en un proyecto a largo plazo para las organizaciones.

5. Modelo de planeación de Fred David. Este modelo consta de tres partes principales:

a) Formulación de estrategias.

b) Ejecución de estrategia.

c) Evaluación de estrategias.

6. Modelo de planeación de Jean Paul Sallenave. Según este autor y corroborado por las reflexiones anteriores reiteramos que la planeación estratégica, es el proceso que sigue el gerente integral para dirigir y controlar el futuro de su empresa, planteándose que en la mayoría de las empresas diversificadas y organizadas según una estructura divisional, la planificación estratégica se establece en tres niveles:

a) A nivel de la empresa total (corporateplanning).Esta responde estas preguntas:

- ¿Dónde invertir los recursos actuales de la empresa?

- ¿De dónde se podrán obtener los recursos futuros?

- ¿Cómo asegurar la supervivencia, el crecimiento y la rentabilidad

b) A nivel de división (business planning). Esta responde a ¿cómo se logrará el éxito en los sectores escogidos?

c) A nivel funcional. Responde a la interrogante, ¿quiénes se responsabilizarán de llevar a cabo el plan estratégico elaborado?

Existen otros modelos en la bibliografía revisada como son los de: Frank Baughart, Tom Lambert, Colon y Rodríguez, Staton y Walter, Ralhf Mroz, etc. Todos muy similares y dan inicio con la formulación de la estrategia.

En la actualidad los gerentes hacen que el resto de la organización participe en el proceso de la formación de estrategias; el ambiente tan cambiante de las tres últimas décadas han obligado a los 
ejecutivos a buscar ideas e innovaciones en todos los niveles de la organización para formar a sus compañías de una manera más competitiva; debido a esta tendencia ha surgido un nuevo término para el proceso de planeación: "administración estratégica". (Cendejas Valdéz, 2014), (Cano Flores \& Olivera Gómez, 2008)

\section{Administración estratégica}

Administración definida por Chiavenato en el año 2001, como, Cito (...): "el proceso de planear, organizar, dirigir y controlar el uso de los recursos para lograr los objetivos"

De acuerdo a este autor, la administración comprende el proceso de diseñar y mantener un ambiente en el que las personas trabajando en grupo alcancen con eficiencias las metas seleccionadas, aplicándose a todo tipo de organización bien sean pequeñas o grandes, lucrativa o no lucrativas, manufactureras o de servicios.

A través de la administración las empresas alcanzan los objetivos trazados que sirven como una guía para la etapa de ejecución de las acciones en forma eficiente, logrando cumplir las metas establecidas con un la utilización mínima de recursos (Velázquez, 2013)

La administración estratégica involucra a los gerentes en la formulación implementación de metas estratégicas y estrategias; quiere decir, que integra la planeación estratégica y administración en un solo proceso, existen muchas posibilidades de que así las organizaciones cambien sus formas de trabajo, lo que le permite ampliar su ventaja.

El nuevo paradigma de gestión/dirección/administración empresarial de los años noventa: la dirección estratégica (strategicmanagement) tiene sus fuentes teóricas y prácticas en la propia planeación estratégica y sus metodologías de análisis.

La gerencia estratégica tiene como función, planteado en el año 1994 por Lambin: Cito (...); “...orientar la empresa hacia oportunidades económicas atractivas para ella (y para la sociedad), es decir, adaptadas a sus recursos y su saber hacer, y que ofrezcan un potencial atrayente de crecimiento y rentabilidad... (Para lo cual deberá) precisar la misión de la empresa, definir sus 
objetivos, elaborar sus estrategias de desarrollo y velar por mantener una estructura racional en su cartera de productos mercados" (Paz Jorge, 2007)

La planeación estratégica exige cuatro fases bien definidas: formulación de objetivos organizacionales; análisis de las fortalezas y limitaciones de la empresa; análisis del entorno; formulación de alternativas estratégicas.

El análisis del entorno está cifrado en identificar las oportunidades que se presentan para la empresa y las amenazas que se vislumbran en el futuro. El análisis interno de la empresa figura en los puntos fuertes y débiles de la organización, por comparación implícita con las demás empresas del sector. Ello tiene que ver con su habilidad distintiva (la fortaleza principal), y se presume que una empresa desarrolla fortalezas en el campo que considera como área estratégica (Castellanos, 2015).

\section{Análisis externo}

Los términos de oportunidad y amenazas hacen referencia a aquellas condiciones favorables o desfavorables para la empresa que derivan de los cambios que se dan en el medio ambiente; entre tales cambios se incluyen tanto las nuevas situaciones que de alguna manera ya están presentes, como los hechos que al futuro pueden verificarse. Existen cuatro procedimientos que llevan a obtener esta información:

1. Explorar el medio ambiente para definir los cambio más significativos que se han dado en los últimos tiempos y los que se pudieran anticipar para el futuro;

2. Indicar qué probabilidad o grado de certeza se atribuye a la ocurrencia de cada uno de esos cambios

3. Establecer qué oportunidades y qué amenazas se abren para la firma si llegan a materializarse dichos cambio; $y$,

4. Finalmente, valorar el nivel de impacto que se tendría. 
La composición de una lista de oportunidades y amenazas debe preparar a la empresa, que recomendándose reducir a un mínimo para poder dirigir la atención a los retos de mayor relevancia. En la figura 1se resumen esta idea.

Figura 1. Análisis externo de una empresa

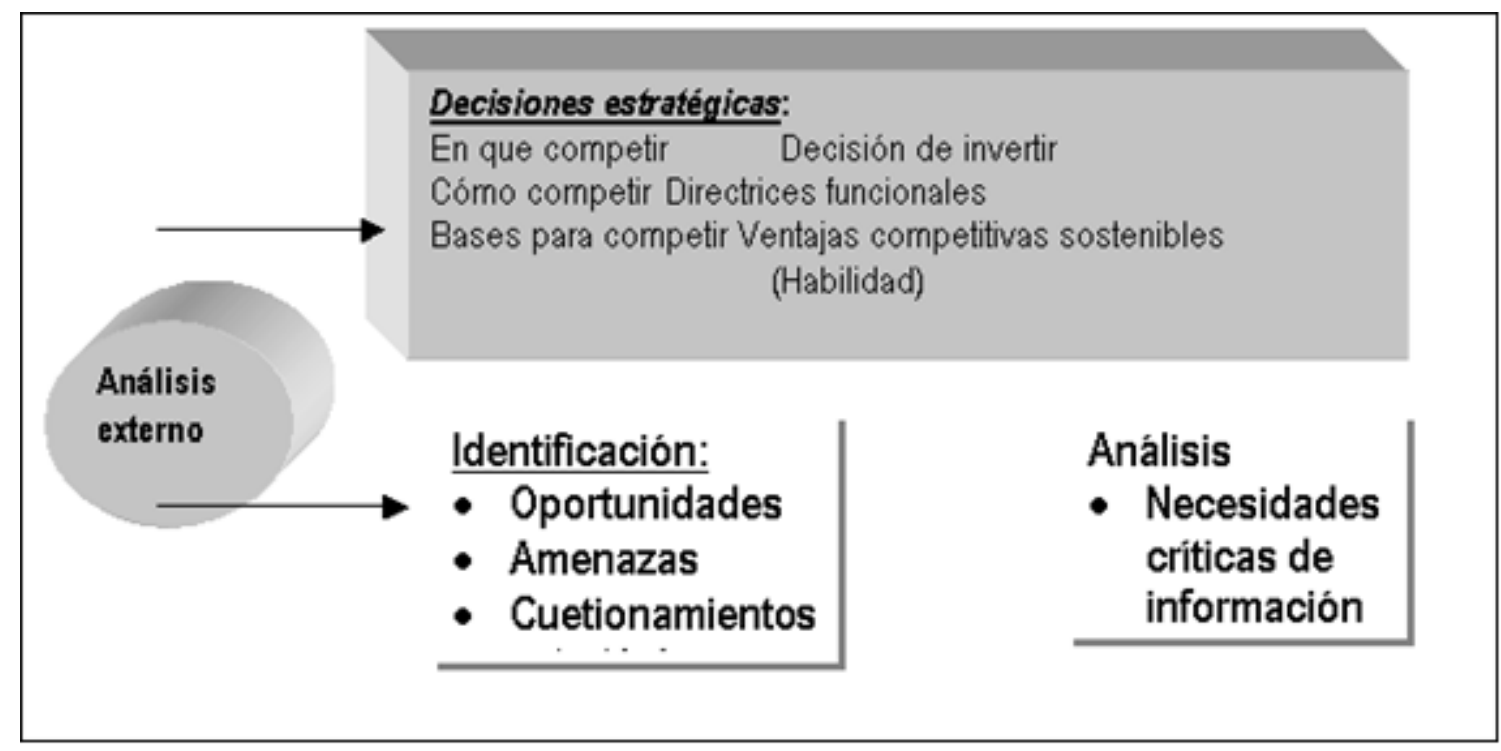

\section{Ejemplos de Decisiones estratégicas}

1- Introducción de nuevos productos

2- Introducción de nuevos procesos

3- Nuevos negocios/empresas

4- Expansión de capacidad instalada

5- Cierre de un negocio/producto

6- Introducción a nuevos mercados

7- Decisiones de desarrollo tecnológico

8- Alianza estratégica

9- Adquisiciones y fusiones

10- Venta de una empresa

11-Entrar al mercado de capitales 
12- Acciones a tomar ante un cambio brusco e inesperado de la paridad de la moneda.

\section{Análisis interno}

Una vez definido las principales oportunidades y amenazas, el trabajo que sigue es valorar los elementos con que se cuenta para ofrecer una respuesta apropiada, lo que clásicamente se ha establecido como fortalezas y debilidades; se deben considerar todos los aspectos que se manejan en la organización, recursos humanos, recursos físicos, recursos financieros, recursos técnicos y tecnológicos, riesgos, etc.,

Las fortalezas se clasifican en:

- Comunes: cuando una fortaleza es poseída por varias empresas o cuando varias están en capacidad de implementarla.

- Distintivas: cuando una misma fortaleza es poseída por un pequeño número de competidores son las que generen ventajas competitivas y desempeños superiores a las del promedio industrial.

- De imitación: son grandes capacidades de copiar y mejorar lasfortalezas distintivas de los demás.

Las debilidades se refieren básicamente a desventajas competitivas, las cuales se presentan cuando no se implementan estrategias generadoras de valor que los competidores sí implementan Se pueden obtener respondiendo a las siguientes preguntas: (Figura 2)

Figura 2. Análisis interno de una empresa 


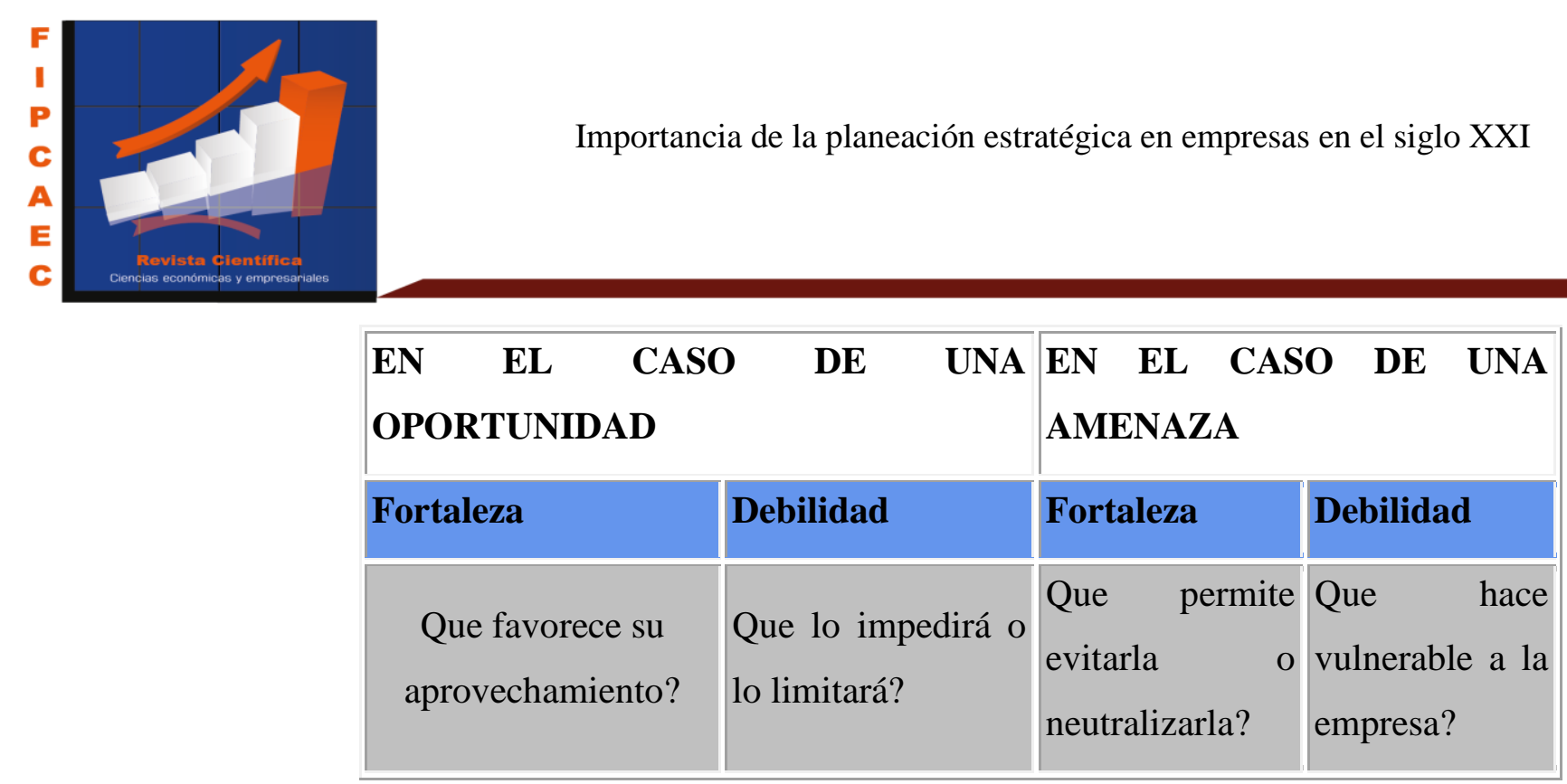

Partiendo de estos cuatro términos o fuerzas se desarrolla la denominada Matriz DAFO, DOFA, FODA o SWOT (por sus siglas en inglés), como quiera llamarse; formada por las iniciales de dichos términos; es una herramienta de múltiple aplicación que puede ser usada por todos los departamentos de la organización en sus diferentes niveles, para analizar diferentes aspectos.

Un análisis DOFA juicioso y ajustado a la realidad provee excelente información para la toma de decisiones en el área de mercados, por ejemplo, permite una mejor perspectiva antes de emprender un nuevo proyecto de producto.

La matriz FODA: Conduce al desarrollo de cuatro tipos de estrategias.

La estrategia FO: Es basa en el uso de fortalezas internas de la organización con el propósito de aprovechas las oportunidades externas. Este tipo de estrategia es el más recomendado. La organización podría partir de sus fortalezas y a través de la utilización de sus capacidades positivas, aprovecharse del mercado para el ofrecimiento de sus bienes y servicios.

La estrategia FA: Trata de disminuir al mínimo el impacto de las amenazas del entorno, valiéndose de las fortalezas. Esto no implica que siempre se deba afrontar las amenazas del entorno de una forma tan directa, ya que a veces puede resultar más problemático para la institución.

La estrategia DA: Tiene como propósito disminuir las debilidades y neutralizar las amenazas, a través de acciones de carácter defensivo. Generalmente este tipo de estrategia se utiliza sólo 
cuando la organización se encuentra en una posición altamente amenazada y posee muchas debilidades, aquí la estrategia va dirigida a la sobrevivencia. En este caso, se puede llegar incluso al cierre de la institución o a un cambio estructural y de misión.

La estrategia DO: Tiene la finalidad mejorar las debilidades internas, aprovechando las oportunidades externas, una organización a la cual el entorno le brinda ciertas oportunidades, pero no las puede aprovechar por sus debilidades, podría decir invertir recursos para desarrollar el área deficiente y así poder aprovechar la oportunidad. (López, 2001) (Lopez Robles, 2015)

El análisis cruzado de las fortalezas y debilidades en relación a las oportunidades y amenazas se ha denominado en la literatura administrativa como "Análisis FODA", el mismo puede ser interpretado en un cuadrante dividido en cuatro secciones 


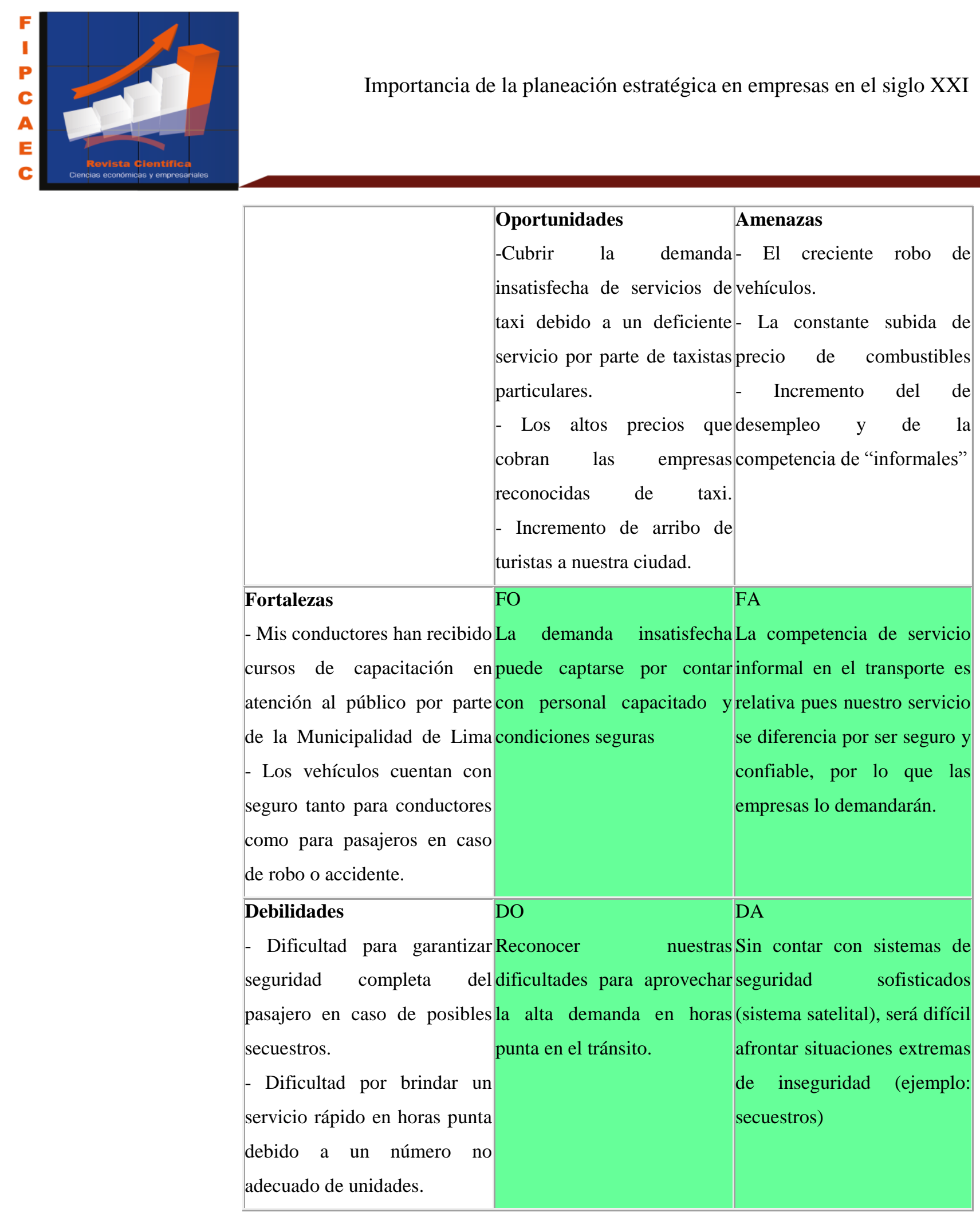

En un cuadrante FO, la empresa posee fortalezas y puede aprovechar las oportunidades que se le presenten; en un cuadrante DO la empresa, puesto que tiene debilidades, se encuentra incapacitada para aprovechar las oportunidades; por su parte, en el cuadrante FA la empresa tiene fortalezas y puede enfrentar con éxito las amenazas que se le presenten; por último, en el 
cuadrante DA la organización se encuentra en posición crítica, pues se le presentan amenazas y ella se encuentra en posición de debilidad. Un análisis de este tipo brinda a los administradores una posibilidad real de evaluar lo que efectivamente puede hacerse (Becerra, 2002).

\section{Proceso de la Planeación estratégica}

El proceso de la administración estratégica consta de tres etapas:
a) Formulación de la estrategia
b) Implementación de la estrategia y
c) Evaluación de la estrategia

\section{Formulación de la Estrategia:}

El primer paso del proceso de la planeación implica la comprensión, confección, redacción y divulgación de la Misión de la organización y después establecer objetivos concretos que nos ayuden a concretar la misión. Esta etapa responde a la pregunta básica que se hacen todos los estrategas ¿qué queremos de la empresa?; La misión expone el porqué de la existencia de la organización y el qué debe hacer, esta misión debe distinguir a la empresa de todas las demás.

La formulación de la estrategia además de elaborar la misión de la empresa, debe detectar las oportunidades y las amenazas externas de la organización, definir sus fuerzas y debilidades, establecer objetivos a largo plazo, generar estrategias alternativas y elegir las estrategias concretas que se seguirán. Algunos aspectos de la formulación de estrategias consisten en decidir en qué nuevos negocios se participará, cuáles se abandonarán, cómo asignar recursos, si es conveniente extender las operaciones o diversificarse, si es aconsejable ingresar a los mercados extranjeros, si es recomendable fusionarse o constituir una empresa de riesgo compartido y cómo evitar una adquisición hostil.

Las elección de las metas u objetivos de la empresa es importante tener en cuenta los valores de los administradores; estos son sociales o éticos, o implicar asuntos prácticos, tales como el 
tamaño que a los administradores les gustaría que tuviera su organización, el tipo de producto o servicio que a ellos les gustaría producir, etc.

\section{Este acápite en el proceso de planeación incluye:}

- Formulación de las metas u objetivos.

- Identificación de los actuales objetivo

- Análisis del ambiente: Análisis externo e interno, ya referidos.

- Toma de decisiones estratégicas donde se generan las alternativas estratégicas o alternativas de solución (Matriz DAFO).

\section{Implementación de la estrategia}

Deben realizarse los siguientes aspectos:

- Diseño de una estructura organizacional

- Diseño de un sistema de control

- Adecuación de la estrategia, la estructura y los controles.

- Manejo de conflictos, las políticas y el cambio.

\section{Evaluación de la estrategia}

1. Otra forma de cómo definir el proceso sería.

2. Definición de la Misión.

3. Definición de la Visión.

4. Análisis de los principales riesgos y oportunidades del entorno social

5. Identificación de fuerzas impulsoras y restrictivas de la organización.

6. Definición de los objetivos estratégicos.

7. Establecimientos de programas y metas de trabajo (Barthelmess Vargas, 2003), (Garcia Guiliany, y otros, 2017) 


\section{Consideraciones finales}

Con la información brindada en esta revisión, una empresa logra la excelencia o el éxito, cuando el gerente con los integrantes de la dirección sean capaces de gestar la planificación estratégica, pero no solo ocuparse de su confección sino de su implementación y control, pues permite a los directivos hacer un plano de verificación de procesos para que los esfuerzos de mejora en estas empresas tengan éxitos, evidenciándose en su competitividad, por lo cual se hace necesario que la mayor parte de los integrantes de la organización estén alineados en un propósito común, formando parte de la visión, misión, valores, aunados a la estrategia organizacional clara y compartida. Isea-Argüelles \& Aldana-Zavala (2017), argumentan que la planeación estratégica es parte fundamental en la mejora continua de la organización, siendo indispensable promover estrategias a partir de la evaluación estratégica como principio primordial en el fomento de evaluaciones con fines de crecer como equipo de trabajo, así mismo, Aldana-Zavala (2017), promueve el enfoque racional como una opción para la generación de planes estratégicos que permitan promover un accionar gerencial transformacional en beneficio de la organización y su competitividad en el mercado global, Aldana \& Piña (2017), comentan que lo planteado conlleva a generar un servicio de calidad al cliente y por ende la organización se revaloriza en el mercado.

\section{Referencias}

1. Aldana, J., \& Piña, J. (2017). Calidad del servicio prestado al cliente por los instructores de gimnasios. Revista Arbitrada Interdisciplinaria Koinonía, 2(3), 172-197. Recuperado de http://fundacionkoinonia.com.ve/ojs/index.php/revistakoinonia/article/view/59/46

2. Aldana-Zavala, J.J. (2017). Modelo de gestión gerencial basado en el modelo racional para la toma de decisiones. Revista Científica FIPCAEC (Fomento De La investigación Y publicación En Ciencias Administrativas, Económicas Y Contables). ISSN: 2588-090X. Polo De Capacitación, Investigación Y Publicación (POCAIP), 2(4), 31-48. https://doi.org/10.23857/fipcaec.v2i4.30 
Económicas Y Contables). ISSN: 2588-090X. Polo De Capacitación, Investigación Y Publicación (POCAIP), 2(2), 17-31. https://doi.org/10.23857/fipcaec.v2i2.15

11. Jackeline, F., \& Posadas, A. (2016). La planeación estratégica en el proceso administrativo. . Obtenido de Gestiopoli: http://www.gestiopolis.com/la-planeacionestrategica-en-el-proceso-administrativo/

12. Lopez Robles, E. (2015). Desarrollo del análisis FODA. . Obtenido de Gestiopoli.: https://www.gestiopolis.com/desarrollo-del-analisis-foda/

13. López, C. (2001). ANÁLISIS DOFA, FODA O SWOT. . Obtenido de Gestiopolis.: http:://gestiopolis.com

14. Paz Jorge, I. (2007). Planeación y la Dirección Estratégica: Futuro de la Empresa Colombiana. . Revista EAFIT(105). Obtenido de http://www.angelfire.com/ca3/blueiguana/herr3.htm

15. Sánchez Jacas, I. (2017). La planeación estratégica en el sistema de salud cubano. $\begin{array}{llll}\text { MEDISAN, } & \text { 21(5). Obtenido }\end{array}$ http://www.medisan.sld.cu/index.php/san/article/view/1011/html

16. Valenzuela Argoti, C. E. (2016). Importancia de la planeación estratégica en las empresas. Tesis Doctoral, Colombia. Obtenido de https://repository.unimilitar.edu.co/bitstream/handle/10654/15416/ValenzuelaArgotiCarlo sErnesto2016.pdf.pdf;jsessionid=EF23477B0A992FD9CA9E28FCE95D6224? sequence= 1

17. Velázquez, I. (2013). Consideraciones teórico conceptuales sobre el proceso de Dirección Estratégica. Capítulo I. . Obtenido de GESTIOPOLI: http://www.gestiopolis.com/diseno-de-un-modelo-de-planificacion-estrategica

\section{References}


9. Garcia Guiliany, J. E., Duran, S. E., Cardeño Pórtela, E., Prieto Pulido, R., Garcia Cali, E., \& Paz Marcano, A. (2017). Strategic Planning Process: Stages executed in small and medium enterprises to optimize competitiveness. Spaces Magazine, 38 (52).

10. Isea-Argüelles, J. \& Aldana-Zavala, J. J. (2017). Kaizen management philosophy aimed at human talent with administrative management responsibilities. FIPCAEC Scientific Magazine (Promotion of research and publication in Administrative, Economic and Accounting Sciences). ISSN: 2588-090X. Training, Research and Publication Center (POCAIP), 2 (2), 17-31. https://doi.org/10.23857/fipcaec.v2i2.15

11. Jackeline, F., \& Posadas, A. (2016). Strategic planning in the administrative process. . Obtained from Gestiopoli: http://www.gestiopolis.com/la-planeacion-estrategica-en-elproceso-administrativo/

12. Lopez Robles, E. (2015). SWOT analysis development. . Obtained from Gestiopoli .: https://www.gestiopolis.com/desarrollo-del-analisis-foda/

13. López, C. (2001). DOFA, SWOT OR SWOT ANALYSIS. . Obtained from Gestiopolis .: http :: //gestiopolis.com

14. Paz Jorge, I. (2007). Planning and Strategic Management: Future of the Colombian Company. . EAFIT Magazine (105). Retrieved from http://www.angelfire.com/ca3/blueiguana/herr3.htm

15. Sánchez Jacas, I. (2017). Strategic planning in the Cuban health system. MEDISAN, 21 (5). Retrieved from http://www.medisan.sld.cu/index.php/san/article/view/1011/html

16. Valenzuela Argoti, C. E. (2016). Importance of strategic planning in companies. Doctoral thesis, Colombia. $\quad$ Retrieved from https://repository.unimilitar.edu.co/bitstream/handle/10654/15416/ValenzuelaArgotiCarlo sErnesto2016.pdf.pdf;jsessionid=EF23477B0A992FD9CA9E28FCE95D6224? sequence= 1 
17. Velázquez, I. (2013). Theoretical conceptual considerations about the Strategic Management process. Chapter I.. Obtained from GESTIOPOLI: http://www.gestiopolis.com/diseno-de-un-modelo-de-planificacion-estrategica 